\title{
P47 - Does vitamin D status in childhood modify risk for asthma development by altering susceptibility to severe respiratory infection and allergic sensitisation?
}

\author{
Elysia Hollams ${ }^{1 *}$, Guicheng Zhang ${ }^{2}$, Barbara Holt ${ }^{1}$, Merci Kusel ${ }^{1}$, Peter Sly ${ }^{3}$, Patrick Holt ${ }^{1}$, Prue Hart ${ }^{1}$ \\ From 3rd Pediatric Allergy and Asthma Meeting (PAAM) \\ Athens, Greece. 17-19 October 2013
}

\section{Background}

Vitamin D has entered the spotlight in the search for preventive treatments against asthma and allergic disease due to its immune-modulating functions, shown in experimental models to include promotion of immune tolerance and boosting protection against infection. Vitamin D inadequacy is common, but disparate findings from cohort studies have had a polarising effect on the scientific community regarding the wisdom of advocating vitamin D supplementation for protection against asthma and allergic disorders. We have previously found that in the high-risk Western Australian CAS cohort (selected due to positive parental atopic history), the combination of multiple severe lower respiratory infections and sensitisation to inhaled allergens by age 2 profoundly increased risk of asthma development by age 5 .

\section{Aims}

To determine whether vitamin $\mathrm{D}$ levels between birth and age 10 years in the CAS cohort are related to frequency of severe respiratory infections in early childhood, allergic sensitisation, and development of asthma by age 5 or 10 years.

\section{Methods}

We used UPLC/MS/MS (accuracy confirmed with DEQAS standards) to measure 25(OH)-vitamin D3, 3-epi25(OH)-vitamin D3 and 25(OH)-vitamin D2 from cryobanked plasma samples collected from CAS participants at birth, then at 6 months and 1, 2, 3, 4, 5 and 10 years. CAS participants were visited by the study physician up to age 5 years for every episode of respiratory infection.

\section{Results}

Vitamin D3 inadequacy was common amongst cohort participants, and as expected was highest amongst participants from whom blood was collected in winter; deseasonalised vitamin D3 was calculated for longitudinal comparisons. In all assessments between 6 months $(n=233)$ and 4 years of age $(n=189)$ the majority of participants had inadequate vitamin D3; 51\%-67\% of participants had insufficient vitamin D3 $(50-75 \mathrm{nmol} / \mathrm{L})$ while 18\%-28\% were vitamin D3 deficient ( $<50 \mathrm{nmol} / \mathrm{L})$. Analyses addressing the aims are underway and will be presented at the Meeting.

\section{Authors' details}

${ }^{1}$ Telethon Institute for Child Health Research, University of Western Australia, Perth, Australia. ${ }^{2}$ University of Western Australia, Perth, Australia. ${ }^{3}$ Queensland Children's Medical Research Institute, Brisbane, Australia.

Published: 28 February 2014 